\title{
Thermal-driven Dynamic Shape Change of Bimetallic Nanoparticles Extends Hot Electron Lifetime of $\mathbf{P t} / \mathrm{MoS}_{2}$ Catalysts
}

\author{
Xiaoli Wang ${ }^{1}$, Run Long ${ }^{1 *}$ \\ ${ }^{1}$ College of Chemistry, Key Laboratory of Theoretical \& Computational
}

Photochemistry of Ministry of Education, Beijing Normal University, Beijing, 100875,

P. R. China

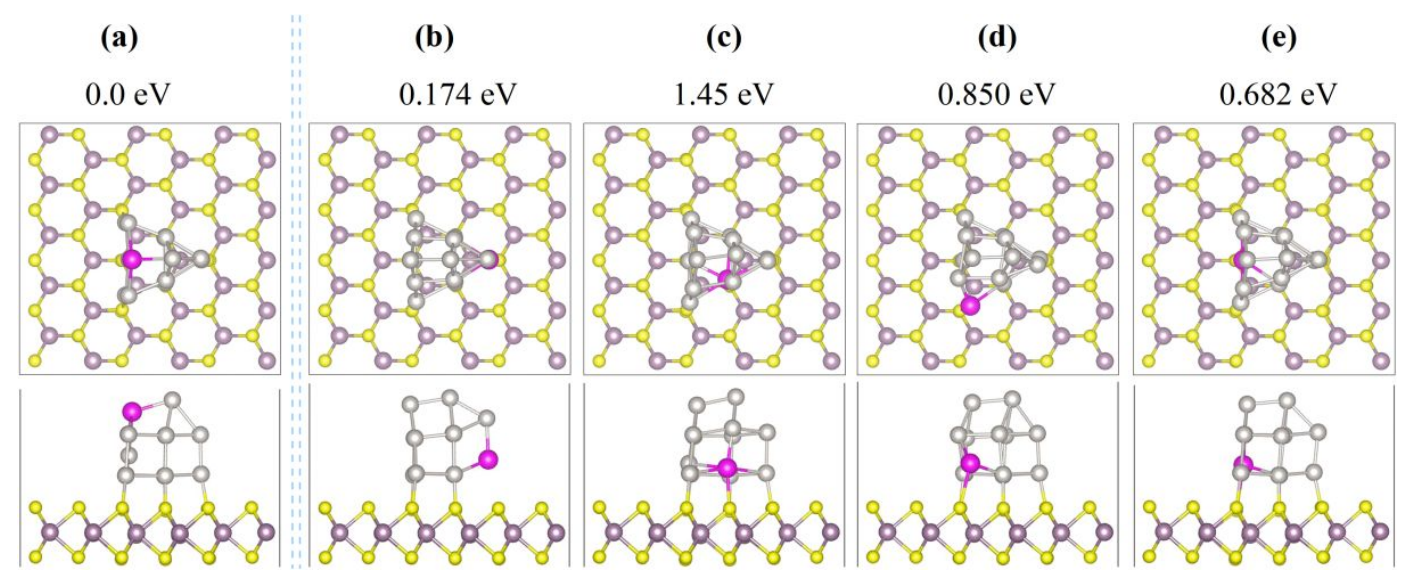

Figure S1. Top and side views of the (a-e) optimized $\mathrm{SnPt}_{12} / \mathrm{MoS}_{2}$ geometries with the substitutional Sn locating at different positions closer to the interface. The relative total energy of (b-e) is $0.174,1.45,0.850$ and $0.682 \mathrm{eV}$, respectively, by setting the total energy of the configuration (a) to $0 \mathrm{eV}$. As a consequence, we chose configuration (a) as the investigated $\mathrm{SnPt}_{12} / \mathrm{MoS}_{2}$ system in the main text.

*Corresponding Author Email: runlong@bnu.edu.cn 
(a)

$6.79 \mathrm{eV}$
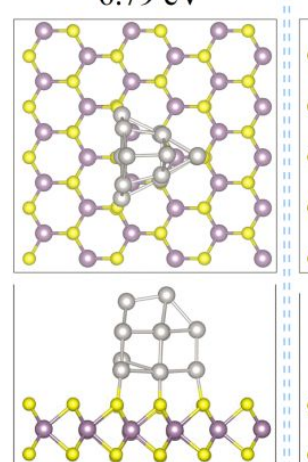

(b)

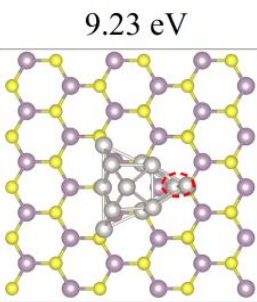

(c)

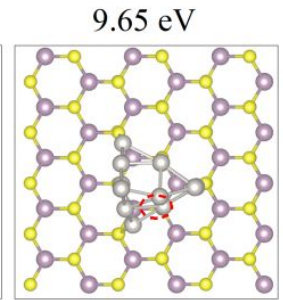

(d)

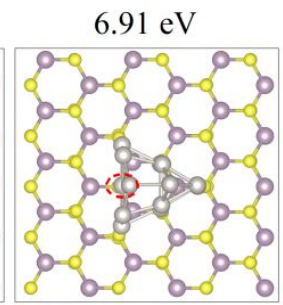

(e)
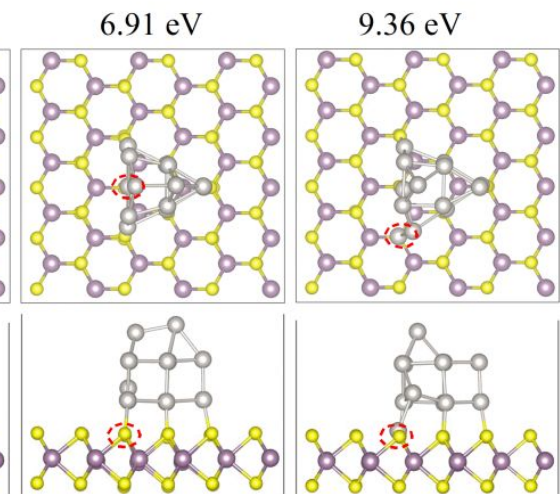

Figure S2. Top and side views of the optimized geometries of (a) pristine $\mathrm{Pt}_{13} / \mathrm{MoS}_{2}$ and (b-e) pristine system with a sulfur vacancy locating at the different interfacial sites. The number above each geometry corresponds to the binding energy between the $\mathrm{Pt}_{13}$ nanoparticle and the $\mathrm{MoS}_{2}$ substrate. The binding energy is calculated according to the formula: $E_{b}=E(A)+E(B)-E(A B)$. Here, $E(A)$ represents the total energy of the optimized $\mathrm{MoS}_{2}$ monolayer with and without a sulfur vacancy, $\mathrm{E}(\mathrm{B})$ represents the total energy of the optimized $\mathrm{Pt}_{13}$ nanoparticle, and $\mathrm{E}(\mathrm{AB})$ represents the total energy of the optimized $\mathrm{Pt}_{13} / \mathrm{MoS}_{2}$ with and without a sulfur vacancy. The value is more positive, and the binding is stronger. The presence of a sulfur vacancy enhances the mutual interaction between the donor and acceptor components. Note, the $\mathrm{Pt}_{13} / \mathrm{MoS}_{2}$ structure presented in the main text corresponds to the geometry shown in configuration (a). 